\title{
Investigation of multiple sclerosis-related pathways through the integration of genomic and proteomic data
}

\author{
Elif Everest ${ }^{1}$, Ege Ülgen ${ }^{2}$, Ugur Uygunoglu ${ }^{3}$, Melih Tutuncu ${ }^{3}$, Sabahattin Saip ${ }^{3}$, Osman Uğur Sezerman ${ }^{2}$, Aksel \\ Siva ${ }^{3}$, Eda Tahir Turanli ${ }^{\text {Corresp. } 1}$ \\ ${ }^{1}$ Department of Molecular Biology and Genetics, Faculty of Science and Letters, Istanbul Technical University, Istanbul, Turkey \\ 2 Department of Biostatistics and Medical Informatics, Faculty of Medicine, Acıbadem University, Istanbul, Turkey \\ 3 Department of Neurology, Cerrahpaşa School of Medicine, Istanbul University-Cerrahpaşa, Istanbul, Turkey \\ Corresponding Author: Eda Tahir Turanli \\ Email address: turanlie@itu.edu.tr
}

Background. Multiple sclerosis (MS) has a complex pathophysiology, variable clinical presentation, and unpredictable prognosis; understanding the underlying mechanisms requires combinatorial approaches that warrant the integration of diverse molecular omics data.

Methods. Here, we combined genomic and proteomic data of the same individuals among a Turkish MS patient group to search for biologically important networks. We previously identified differentiallyexpressed proteins by cerebrospinal fluid proteome analysis of 179 MS patients and 42 non-MS controls. Among this study group, 11 unrelated MS patients and 60 independent, healthy controls were subjected to whole-genome SNP genotyping, and genome-wide associations were assessed. Pathway enrichment analyses of MS-associated SNPS and differentially-expressed proteins were conducted using the functional enrichment tool, PANOGA.

Results. Nine shared pathways were detected between the genomic and proteomic datasets after merging and clustering the enriched pathways. Complement and coagulation cascade was the most significantly associated pathway ( $\mathrm{hsa} 04610, \mathrm{P}=6.96 \times 10^{-30}$ ). Other pathways involved in neurological or immunological mechanisms included adherens junctions (hsa04520, $\mathrm{P}=6.64 \times 10^{-25}$ ), pathogenic Escherichia coli infection (hsa05130, $\mathrm{P}=9.03 \times 10^{-14}$ ), prion diseases (hsa05020, $\mathrm{P}=5.13 \times 10^{-13}$ ).

Conclusion. We conclude that integrating multiple datasets of the same patients helps reducing false negative and positive results of genome-wide SNP associations and highlights the most prominent cellular players among the complex pathophysiological mechanisms. 


\section{Investigation of multiple sclerosis-related pathways through 2 the integration of genomic and proteomic data}

3

4 Elif Everest ${ }^{1}$, Ege Ülgen ${ }^{2}$, Ugur Uygunoglu ${ }^{3}$, Melih Tutuncu ${ }^{3}$, Sabahattin Saip ${ }^{3}$, Osman Uğur

5 Sezerman $^{2}$, Aksel Siva $^{3}$, Eda Tahir Turanli ${ }^{1}$

6

7

8

${ }^{1}$ Department of Molecular Biology and Genetics, Faculty of Science and Letters, Istanbul Technical University, Istanbul, Turkey

${ }^{2}$ Department of Biostatistics and Medical Informatics, Faculty of Medicine, Acibadem University, Istanbul, Turkey

${ }^{3}$ Department of Neurology, Cerrahpaşa School of Medicine, Istanbul University-Cerrahpaşa, Istanbul, Turkey

Corresponding Author:

Eda Tahir Turanli ${ }^{1}$ Istanbul Technical University, Ayazağa Campus, Faculty of Science and Letters, Department of Molecular Biology and Genetics, Istanbul, 34469, Turkey
Email address: turanlie@itu.edu.tr 
38 Abstract

39 Background. Multiple sclerosis (MS) has a complex pathophysiology, variable clinical

40 presentation, and unpredictable prognosis; understanding the underlying mechanisms requires

41 combinatorial approaches that warrant the integration of diverse molecular omics data.

42 Methods. Here, we combined genomic and proteomic data of the same individuals among a

43 Turkish MS patient group to search for biologically important networks. We previously

44 identified differentially-expressed proteins by cerebrospinal fluid proteome analysis of $179 \mathrm{MS}$

45 patients and 42 non-MS controls. Among this study group, 11 unrelated MS patients and 60

46 independent, healthy controls were subjected to whole-genome SNP genotyping, and genome-

47 wide associations were assessed. Pathway enrichment analyses of MS-associated SNPs and

48 differentially-expressed proteins were conducted using the functional enrichment tool,

49 PANOGA.

50 Results. Nine shared pathways were detected between the genomic and proteomic datasets after 51 merging and clustering the enriched pathways. Complement and coagulation cascade was the 52 most significantly associated pathway (hsa04610, $\mathrm{P}=6.96 \times 10^{-30}$ ). Other pathways involved in 53 neurological or immunological mechanisms included adherens junctions (hsa04520, $\mathrm{P}=6.64 \times$

$541^{-25}$ ), pathogenic Escherichia coli infection (hsa05130, $\mathrm{P}=9.03 \times 10^{-14}$ ), prion diseases 55 (hsa05020, $\left.\mathrm{P}=5.13 \times 10^{-13}\right)$.

56 Conclusion. We conclude that integrating multiple datasets of the same patients helps reducing 57 false negative and positive results of genome-wide SNP associations and highlights the most 58 prominent cellular players among the complex pathophysiological mechanisms.

59

60

61

62

63

\section{Introduction}

MS is known to be an immune-mediated, neurodegenerative CNS disorder with complex inheritance and pathophysiological mechanisms. Although approximately common 250 genetic variants with low to modest risk effects have been associated with MS mainly by genome-wide association studies (GWAS) using rather large sample groups (International Multiple Sclerosis Genetics Consortium, 2007; Sawcer et al., 2011; Patsopoulos et al., 2017; International Multiple Sclerosis Genetics Consortium et al., 2019), known variants not only fail to explain predicted MS heritability but also cannot be efficiently translated into disease mechanisms. To date, numerous studies have also revealed potential diagnostic and prognostic biomarkers and diseaserelated cellular pathways that emphasize the different pathological components of the disease; however, the exact underlying mechanisms in disease development and progression are largely unknown. In order to better translate the growing number of findings into disease pathophysiology, algorithms for pathway analyses of multiple high-throughput omics data seem essential. In this context, the integration of multiple omics data is essential to better describe the complex nature of MS.

In our previous study (Avsar et al., 2015), we have conducted a cerebrospinal fluid (CSF) proteome analysis using 2D-gel electrophoresis and mass spectrophotometry and identified 151 differentially expressed proteins between an MS cohort of 179 patients with different clinical MS 
78 phenotypes and 42 non-MS controls. Later, affected Kyoto Encyclopedia of Genes and Genomes

79 (KEGG) pathways were identified using the functional enrichment tool Pathway and Network-

80 Oriented GWAS Analysis (PANOGA) (Bakir-Gungor, Egemen \& Sezerman, 2014), revealing

81 MS-related pathways including aldosterone-regulated sodium reabsorption pathway, renin-

82 angiotensin system, notch signaling pathway, and vitamin digestion and absorption pathway.

83 Here, we further explored disease-related pathways, applying single nucleotide polymorphism

84 (SNP) genotyping on $11 \mathrm{MS}$ patients, who were included in the previous proteomic study, and

8560 independent, healthy individuals. Pathway enrichment analyses of the genomic and proteomic

86 data were conducted. The two datasets were merged, highlighting the most prominent pathways

87 that may be affected in the studied patient group.

88

89 Materials \& Methods

90 Study groups

91 All patients were diagnosed at Istanbul University-Cerrahpaşa, Cerrahpaşa School of Medicine,

92 Department of Neurology, according to the McDonald criteria (Polman et al., 2011). Eleven

93 unrelated MS patients were randomly selected among the study group of our previous work

94 comprising of $179 \mathrm{MS}$ patients. The patient group had a heterogeneous disease presentation at

95 the time of their CSF sample collection. Sixty independent age- and gender-matched intrinsic

96 healthy controls were included in the analyses. All individuals in the study group are of Turkish

97 origin. All procedures of the study were in accordance with the Helsinki Declaration of 1964 and

98 its later amendments. The Ethics Committee of Istanbul Technical University approved the study

99 (İTÜ-SM.İNAREK-MBG-1), and each individual of the study group gave a written informed

100 consent form prior to the sample collection.

101 Genome-wide associations

102 DNA isolation from blood samples was performed (Roche DNA Isolation Kit for Mammalian

103 Blood), and genotyping for 300.000 SNP markers was applied for each individual on the

104 Illumina HumanCytoSNP-12 array. Illumina GenomeStudio software was used for quality

105 control. All individuals showed sample call rates of more than 98\% (98.65-99.63\%) and were

106 therefore included in the study. Golden Helix SNP \& Variation Suite software was used for

107 identity-by-descent detection, suggesting no relatedness between the individuals. SNP filtering

108 was performed: SNPs on the Y chromosome, with call rates lower than $95 \%$, minor allele

109 frequency lower than 0.01 , and in strong linkage disequilibrium $\left(\mathrm{r}^{2}>0.5\right)$ were excluded from

110 the study. A total of 129.547 SNPs was included in the analysis. Frequency differences of SNPs

111 between cases and controls were assessed, and genotypic association P-values were obtained.

112 Pathway enrichment analysis

113 Genotypic associations and proteomic data were subjected to pathway enrichment analyses using

114 the functional enrichment tool PANOGA, which reveals functionally relevant pathways by

115 identifying genes within the pathways, incorporating protein-protein interaction (PPI)

116 information, and extracting significant pathways. SNPs with genotypic association P-values

117 lower than 0.05 and differentially-expressed proteins with P-values lower than 0.05 from our 
118 previous study were used for PANOGA procedure as follows: For SNP associations, because a

119 SNP might affect more than one gene, each SNP was initially assigned to the gene on which the 120 SNP has the most important functional effect, using the SPOT webserver (Saccone et al., 2010). 121 Functional information of SNPs was obtained utilizing SPOT, F-SNP (Lee \& Shatkay, 2007), 122 SNPnexus (Dayem Ullah, Lemoine \& Chelala, 2012), and SNPinfo (Xu \& Taylor, 2009). Genes 123 and proteins were then mapped onto a PPI network, for which Goh et al.'s human PPI network

124

125

126

127

128

129

130

131

132

133

134

135

136

137

138

139

140

141

142

143

144

145

146

147

148

149

150

151

152

153

154

155

156 was used (Goh et al., 2007). Next, the jActive Modules algorithm (Ideker et al., 2002) was applied to identify active subnetworks containing a large number of the disease-affected genes and proteins in the PPI network. Each genotypic association and differential expression P-value was taken into account, and active subnetworks that overlap at most 50\% with each other were extracted. In order to evaluate the biological importance of the subnetworks, the number of genes and proteins found in a specific KEGG pathway was compared to the total gene and protein number involved in the corresponding pathway. For this functional enrichment procedure, a twosided hypergeometric test was used, and the Bonferroni method was applied for multiple testing corrections of the P-values. Significantly associated KEGG pathways to our patient group consisted of significantly enriched $(\mathrm{P}<0.05)$ pathways for at least one of the active subnetworks. For the pathways that were enriched in multiple subnetworks, the one with the lowest P-value was reported. PANOGA was run 10 times for both genotypic associations and differentiallyexpressed proteins, and the lowest P-values over the 10 iterations were reported. The resulting enriched pathways for both datasets were then merged. If a given pathway was identified in both analyses, the corresponding P-values were merged using Fisher's combined probability test. If the pathway was identified in only one of the analyses, the corresponding P-value was used as the final P-value.

\section{Clustering of enriched pathways}

Using a method previously described by Chen et al., we clustered the enriched pathways to identify similar groups and establish representative pathways (Chen et al., 2014). The clustering approach can be summarized as follows: initially, the overlap index matrix (OI) that consists of the overlap indices between all of the pairs of enriched pathways was calculated. The overlap index $\left(O I_{i, j}\right)$ of a pair of pathways $P_{i}$ and $P_{j}$ (denoting the $i^{\text {th }}$ and $j^{\text {th }}$ pathways, respectively) was defined as:

$$
O I_{i, j}=\frac{\left|G_{i} \cap G_{j}\right|}{\min \left(\left|G_{i}\right|,\left|G_{j}\right|\right)}
$$

where $G_{i}$ is the set of all genes in the $i^{t h}$ pathway. In the overlap matrix, each row $\left(o_{i}\right)$ represents the gene overlap profile of a pathway (i.e., $o_{i}$ is the gene overlap profile of the $i^{t h}$ pathway). To identify similarity between each pair of pathways, the Pearson correlation coefficients (R) between each pair of overlap profiles (e.g., $o_{i}$ and $o_{j}$ ) were calculated. These correlation coefficients were then converted to pairwise distances (PD) as:

$$
P D=1-R
$$

Using PD as the distance metric, hierarchical clustering (average-linkage) of the enriched pathways was performed. Examining the hierarchical clustering dendrogram, the dendrogram 
157 was partitioned into clusters at the manually selected PD cut-off value of 0.55 . The pathway with

158

159

160

161

162

163

164

165

166

167

168

169

170

171

172

173

174

175

176

177

178

179

180

181

182

183

184

185

186

187

188

189

190

191

192

193

194

195

196

the lowest P-value in each cluster was selected as the representative pathway for that cluster.

\section{Results}

\section{SNP associations}

All 71 individuals and a total of 129.547 SNPs passed the quality check and therefore were included in the final analyses. The Manhattan plot summarizing the genome-wide associations is given in Fig. 1. The most significantly associated SNP was rs7873, located on 3' UTR of the $I G F 2$ gene, whose significance level was the only value close to a classical stringent P-value cutoff for a GWAS $\left(\mathrm{P}=4.39 \times 10^{-07}\right)$. When considering SNPs with a significance level of lower than a looser P-value cut off $\left(\mathrm{P}<10^{-05}\right)$ given the small sample size, 4 more SNPs have high associations with MS in the studied patient group, one of which is located on a gene: rs 17187282 $\left(\mathrm{P}=1.17 \times 10^{-06}\right), \mathrm{rs} 11688088\left(\mathrm{P}=2.8 \times 10^{-06}\right), \mathrm{rs} 654188\left(\mathrm{P}=5.24 \times 10^{-06}\right)$, and $\mathrm{rs} 7092208(\mathrm{P}=$ $9.66 \times 10^{-06}$, intronic variant on the $M G M T$ gene).

For enrichment analysis of the SNP associations, the P-value threshold was set lower than 0.05

(Fig. 1, blue line) in order to prevent the elimination of potentially MS-associated SNPs with falsely high P-values due to the low sample size and limitations of the genome-wide association methodology. Later, elimination of the false-positive SNP associations resulted from preferring not to set a conventional stringent $\mathrm{P}$-value was aimed to be achieved during the identification of MS-related pathways using SNP subnetworks, therefore ruling functionally irrelevant SNPs out. A total of 6.594 SNPs had P-values under the threshold of 0.05 (Table S1), which were included in the subsequent analyses.

\section{MS-related pathways}

The combination of enriched pathways for the resulting SNPs and differentially expressed proteins resulted in 151 enriched pathways in total (Table S2). In order to identify pathways with similar content and function, this combined list of enriched pathways was clustered (Fig. 2). The dendrogram was then manually partitioned into biologically relevant clusters, and representative pathways were established. In total, 33 clusters, therefore 33 representative pathways were obtained (Table S3). Nine of the representative pathways emerged from both genomic and proteomic analyses, among which the complement and coagulation cascade (hsa04610) was the most significantly associated pathway in the studied group $\left(\mathrm{P}=6.96 \times 10^{-30}\right)$. Figure 3 shows the complement and coagulation cascades with genes identified through the genomic dataset and differentially expressed proteins identified through the proteomic dataset as a representative pathway for the relationships between the genomic and proteomic findings.

Regulation of actin cytoskeleton (hsa04810) and focal adhesions (hsa04510) were the two systems with the second and third lowest $\mathrm{P}$-values even though there were no significant protein level changes related to these pathways $\left(\mathrm{P}=9.64 \times 10^{-27}\right.$ and $\mathrm{P}=3.29 \times 10^{-26}$, respectively). Other 8 shared pathways with high significance levels are as follows: Adherens junctions (hsa04520, $\mathrm{P}=5.38 \times 10^{-17}$ ), colorectal cancer (hsa05210, $\mathrm{P}=1.70 \times 10^{-15}$ ), pathogenic Escherichia coli infection (hsa05130, P $=7.02 \times 10^{-9}$ ), prion diseases (hsa05020, $\mathrm{P}=2.48 \times 10^{-}$ 
$197{ }^{5}$ ), endometrial cancer (hsa05213, $\mathrm{P}=6.00 \times 10^{-9}$ ), non-small cell lung cancer (hsa05223, $\mathrm{P}=$ $1981.40 \times 10^{-8}$ ), bladder cancer (hsa05219, $\mathrm{P}=4.83 \times 10^{-6}$ ), and non-homologous end-joining 199 (hsa03450, $\mathrm{P}=4.27 \times 10^{-5}$ ). Table 1 shows the detected SNP associations and differentially 200 expressed proteins in the above-mentioned pathways.

201 The most significantly MS-associated pathways obtained from the SNP associations and 202 differentially expressed proteins did not overlap when the analyses were performed separately 203 (Table S2). The merged data analysis resulted in a combined list of pathways from both datasets, 204 revealing a different set of pathways with the lowest P-values and emphasizing the most relevant 205 pathways that could not have been prioritized by a straight-forward, non-combinatorial omics

206

207

208

209

210

211

212

213

214

215

216

217

218

219

220

221

222

223

224

225

226

227

228

229

230

231

232

233

234

235

236 approach. The top 5 pathways that emerged from the proteomic-only, genomic-only, and merged dataset analyses are given in Table 2 .

\section{Discussion}

The complement and coagulation cascade (CCC) pathway emerged as the most significantly associated pathway to MS in our patient group, with many SNP associations on different genes and differentially expressed CSF proteins as shown in Figure 3. The complement and the coagulation systems are two closely linked cascades, both having roles in immunity. In a study by Magliozzi et al. (2019), CSF proteomic profiles of MS patients with low or high cortical lesion load were compared, revealing that the identified differentially expressed proteins were mainly involved in the complement and coagulation cascade. Examination of white matter lesions of MS patients has revealed dysregulation of coagulation-associated proteins in chronic active plaques involving Serpin A5 and tissue factor (Han et al., 2008). Also, significantly upregulated Serpin E1, another CCC component, was reported in the post-mortem cortex of progressive MS patients (Yates et al., 2017). In our study, even though the genes and differentially expressed proteins involved in CCC do not overlap, the pathway was found 10 times for each dataset in PANOGA, resulting in a high association with MS. Our results confirm the previous findings suggesting the involvement of CCC alterations in MS pathophysiology and highlight other pathway components that may also be responsible for these alterations.

Disruption of blood-brain barrier (BBB) integrity is one of the hallmarks in MS pathophysiology, during which massive leukocyte infiltration occurs across the damaged BBB into the CNS (Alvarez, Cayrol \& Prat, 2011). Tight and adherens junctions between the endothelial cells of BBB have significant importance for normal immune surveillance in the CNS. Tight junctions consist of claudins, occludin, junctional adhesion molecules, and Zonula Occludens (Hawkins \& Davis, 2005), whose dysfunctions leading to BBB abnormalities in MS have been previously reported (Kirk et al., 2003; Padden et al., 2007; Plumb et al., 2002). Cadherins form adherens junctions via homophilic interactions between endothelial cells and bind cytoskeletal components through cytoplasmic catenin proteins (Dejana, Orsenigo \& Lampugnani, 2008). Although adherens junctions are required for the overall junctional organization to maintain the BBB integrity, their roles in normal physiology and diseased states have not been wellestablished. In a previous study, the level of an adherens junction protein, $\beta$-catenin, was similar 
237 in progressive MS and non-MS brain sections (Padden et al., 2007). In our patient group, 238 adherens junctions, which were not detected in our previous proteomic study, emerged as the

239

240

241

242

243

244

245

246

247

248

249

250

251

252

253

254

255

256

257

258

259

260

261

262

263

264

265

266

267

268

269

270

271

272

273

274

275

276 second most significantly altered cellular components with SNP associations on different genes and differentially expressed proteins indirectly related to the pathway. Our findings indicate that adherens junctions may have more influence on BBB function than it has been thought and are needed to be explored in both normal physiology and MS pathophysiology in more detail. Focal adhesions and actin cytoskeleton regulation are two interrelated pathways that emerged with low final P-values from the analyses, even though no significant protein expression changes directly or indirectly related to these systems were detected. Organized clusters of focal adhesion complexes connect extracellular matrix through transmembrane integrin proteins to the intracellular actin cytoskeleton through other focal adhesion proteins in the complexes and mainly have roles in cell motility (Wozniak et al., 2004). During leukocyte infiltration across the BBB, $\alpha 4$-integrin proteins on leukocytes form firm connections with the endothelial surface, initiating the process (Berlin et al., 1995; Vajkoczy, Laschinger \& Engelhardt, 2001). In this context, Natalizumab (Antegren, Elan Pharmaceuticals and Biogen), a monoclonal antibody against $\alpha 4$-integrin, has been used in MS treatment, which suppresses inflammatory activity by inhibiting leukocyte migration to the inflammation areas (Polman et al., 2006). We detected many SNP associations on genes encoding for many focal adhesion proteins, including the $\alpha 4-$ integrin-coding ITGA4 gene. Focal adhesion molecules and their combinations to create different complexes are diverse, and regulation differences of these interactions for specific cell behaviors are yet to be elucidated.

In the studied MS patients, increased CSF nucleolin expression and SNP associations on WASL and ROCK2 genes were detected, all of which are components of the Pathogenic Escherichia coli infection pathway. Neural Wiskott-Aldrich syndrome protein encoded by WASL and Rhoassociated protein kinase 2 encoded by ROCK2 are both regulators of the actin cytoskeleton (Miki, Miura \& Takenawa, 1996; Gallo et al., 2012), and the involvement of the pathway may indicate induction of inflammatory cell mobility. Prion diseases are fatal conditions presenting neuronal degeneration, and the emergence of the pathway from our analyses emphasizes some shared molecular alterations with MS pathophysiology, as both neurodegeneration and neuroinflammation also occur in prion diseases (Perry, Cunningham \& Boche, 2002; Eikelenboom et al., 2002). We have also detected a number of cancer pathways involving both SNP associations and differential CSF expressions. Cancer risk among MS patients has been investigated in a number of studies with diverse results, revealing unchanged (Nielsen et al., 2006), reduced (Bahmanyar et al., 2009), and increased (Grytten et al., 2020) cancer rates. Altered cancer pathways in our study group may be attributed to changes in immunological mechanisms involving the same components as in mechanisms against anti-tumor surveillance. Other pathways that emerged only from the SNP associations included a number of immunological and neurological mechanisms. T cell receptor signaling pathway (hsa04660, $\mathrm{P}=$ $1.86 \times 10^{-19}$ ), chemokine signaling pathway (hsa04062, $\mathrm{P}=4.38 \times 10^{-19}$ ), and Fc-gamma Rmediated phagocytosis (hsa04666, $\mathrm{P}=8.78 \times 10^{-16}$ ) were the immune system-related pathways. 
277 Nervous system-related pathways included axon guidance $\left(\right.$ hsa04360, $\left.\mathrm{P}=6.36 \times 10^{-20}\right)$, 278 retrograde endocannabinoid signaling ( $\left.\mathrm{hsa} 04723, \mathrm{P}=6.74 \times 10^{-18}\right)$, neurotrophin signaling 279 pathway (hsa04722, $\left.\mathrm{P}=8.03 \times 10^{-18}\right)$, glutamatergic synapse $\left(\mathrm{hsa} 04724, \mathrm{P}=1.74 \times 10^{-15}\right)$, 280 cholinergic synapse (hsa04725, $\mathrm{P}=7.79 \times 10^{-15}$ ), and dopaminergic synapse (hsa04728, $\mathrm{P}=5.83$ $281 \times 10^{-12}$ ). Also, the Rap1 signaling pathway (hsa04015, $\mathrm{P}=2.35 \times 10^{-20}$ ) was detected, further 282 supporting the involvement of adherens junction, focal adhesion, and regulation of actin 283 cytoskeleton pathways.

284 Working on small sample sizes for genome-wide associations leads to enormous numbers of 285 false-positive SNP associations. Using large sample sizes, on the other hand, may result in false286 negative results due to the low effect sizes of disease-associated SNPs. One limitation of our 287 study is the considerably low sample size in the assessment of SNP associations. In this regard, 288 we aimed to take advantage of having both DNA and CSF samples of the same affected

289

290

291

292

293

294

295

296

297

298

299

300

301

302

303

304

305

306

307

308

309

310

311

\section{References}

313

314

315

\section{Conclusions} individuals. Therefore, we aimed to include both false-positive SNP associations and prevent missing out SNPs with falsely high P-values in the first place by setting a non-stringent P-value cut-off. We then aimed to exclude false-positive associations during the pathway enrichment analyses in which interacting SNP subnetworks were used to identify the pathways, eliminating functionally irrelevant SNPs. Integrative analyses of the genomic and proteomic datasets determined the shared pathways between the two datasets, which seem to represent the pathophysiological mechanisms. Another limitation of the study is that we have used a genotyping chip with a relatively low SNP number $(300 \mathrm{~K})$ since it was one of the most powerful chips when the genotyping had been carried out. Lastly, we had not obtained mRNA samples of the study participants at the time of their sample collection, which would lead to a more comprehensive analysis of the altered pathways in the studied patient group.

Here, we provide an integrated pathway analysis of whole-genome SNP and CSF proteome datasets obtained from the same MS patients, highlighting the most prominent molecular pathways that may be altered in the studied patient group. We confirmed the involvement of some of the previously known players in MS pathophysiology and suggested possible roles of some others. We conclude that this approach provides a better placing of separate genomic and proteomic datasets into a biological context and is useful for elucidating disease mechanisms.

\section{Acknowledgements}

We thank the patients and volunteers who agreed to contribute to the study.

Alvarez JI, Cayrol R, Prat A. 2011. Disruption of central nervous system barriers in multiple sclerosis. Biochimica et Biophysica Acta (BBA)-Molecular Basis of Disease. 1812 (2): 252-64. doi: 10.1016/j.bbadis.2010.06.017. 
316 Avsar T, Durası İM, Uygunoğlu U, Tütüncü M, Demirci NO, Saip S, Sezerman OU, Siva A, 317 Turanlı ET. 2015. CSF proteomics identifies specific and shared pathways for multiple sclerosis 318 clinical subtypes. PloS one. 10 (5): e0122045. doi: 10.1371/journal.pone.0122045.

319 Bahmanyar S, Montgomery SM, Hillert J, Ekbom A, Olsson T. 2009. Cancer risk among patients

320

321

322

323

324

325

326

327

328

329

330

331

332

333

334

335

336

337

338

339

340

341

342

343

344

345

346

347

348

349

350

351

352

353

354 with multiple sclerosis and their parents. Neurology. 72 (13): 1170-7. doi: 10.1212/01.wnl.0000345366.10455.62.

Bakir-Gungor B, Egemen E, Sezerman OU. 2014. PANOGA: a web server for identification of SNP-targeted pathways from genome-wide association study data. Bioinformatics. 30 (9): 12879. doi: 10.1093/bioinformatics/btt743.

Berlin CB, Bargatze RF, Campbell JJ, Von Andrian UH, Szabo MC, Hasslen SR, Nelson RD, Berg EL, Erlandsen SL, Butcher EC. 1995. $\alpha 4$ integrins mediate lymphocyte attachment and rolling under physiologic flow. Cell. 80 (3): 413-22. doi: 10.1016/0092-8674(95)90491-3. Chen YA, Tripathi LP, Dessailly BH, Nyström-Persson J, Ahmad S, Mizuguchi K. 2014. Integrated pathway clusters with coherent biological themes for target prioritisation. PloS one. 9 (6): e99030. doi: 10.1371/journal.pone.0099030.

Dayem Ullah AZ, Lemoine NR, Chelala C. 2012. SNPnexus: a web server for functional annotation of novel and publicly known genetic variants (2012 update). Nucleic acids research. 40 (W1): W65-70. doi: 10.1093/nar/gks364.

Dejana E, Orsenigo F, Lampugnani MG. 2008. The role of adherens junctions and VE-cadherin in the control of vascular permeability. Journal of cell science. 121 (13): 2115-22. doi: 10.1242/jcs.017897.

Eikelenboom P, Bate C, Van Gool WA, Hoozemans JJ, Rozemuller JM, Veerhuis R, Williams A. 2002. Neuroinflammation in Alzheimer's disease and prion disease. Glia. 40 (2): 232-9. doi: 10.1002/glia.10146.

Gallo RM, Khan MA, Shi J, Kapur R, Wei L, Bailey JC, Liu J, Brutkiewicz RR. 2012. Regulation of the actin cytoskeleton by Rho kinase controls antigen presentation by CD1d. The Journal of Immunology. 189 (4): 1689-98. doi: 10.4049/jimmunol.1101484.

Goh KI, Cusick ME, Valle D, Childs B, Vidal M, Barabási AL. 2007. The human disease network. Proceedings of the National Academy of Sciences. 104 (21): 8685-90. doi: 10.1073/pnas.0701361104.

Grytten N, Myhr KM, Celius EG, Benjaminsen E, Kampman M, Midgard R, Vatne A, Aarseth JH, Riise T, Torkildsen Ø. 2020. Risk of cancer among multiple sclerosis patients, siblings, and population controls: A prospective cohort study. Multiple Sclerosis Journal. 26 (12): 1569-80. doi: $10.1177 / 1352458519877244$. Han MH, Hwang SI, Roy DB, Lundgren DH, Price JV, Ousman SS, Fernald GH, Gerlitz B, Robinson WH, Baranzini SE, Grinnell BW. 2008. Proteomic analysis of active multiple sclerosis lesions reveals therapeutic targets. Nature. 451 (7182): 1076-81. doi: 10.1038/nature06559. Hawkins BT, Davis TP. 2005. The blood-brain barrier/neurovascular unit in health and disease. Pharmacological reviews. 57 (2): 173-85. doi: 10.1124/pr.57.2.4. 
355

356

357

358

359

360

361

362

363

364

365

366

367

368

369

370

371

372

373

374

375

376

377

378

379

380

381

382

383

384

385

386

387

388

389

390

391

392

Ideker T, Ozier O, Schwikowski B, Siegel AF. 2002. Discovering regulatory and signalling circuits in molecular interaction networks. Bioinformatics. 18 (suppl_1): S233-40. doi: 10.1093/bioinformatics/18.supp1_1.s233.

International Multiple Sclerosis Genetics Consortium. 2007. Risk alleles for multiple sclerosis identified by a genomewide study. New England Journal of Medicine. 357 (9): 851-62. doi:10.1056/NEJMoa073493.

International Multiple Sclerosis Genetics Consortium*†, ANZgene, IIBDGC, WTCCC2. 2019.

Multiple sclerosis genomic map implicates peripheral immune cells and microglia in susceptibility. Science. 365 (6460): eaav7188. doi: 10.1126/science.aav7188.

Kanehisa M, Goto S. 2000. KEGG: Kyoto Encyclopedia of Genes and Genomes. Nucleic Acids Research. 28 (1): 27-30. doi: 10.1093/nar/28.1.27.

Kirk J, Plumb J, Mirakhur M, McQuaid S. 2003. Tight junctional abnormality in multiple sclerosis white matter affects all calibres of vessel and is associated with blood-brain barrier leakage and active demyelination. The Journal of Pathology: A Journal of the Pathological Society of Great Britain and Ireland. 201 (2): 319-27. doi: 10.1002/path.1434.

Lee PH, Shatkay H. 2007. F-SNP: computationally predicted functional SNPs for disease association studies. Nucleic acids research. 36 (suppl_1): D820-4. doi: 10.1093/nar/gkm904. Magliozzi R, Hametner S, Facchiano F, Marastoni D, Rossi S, Castellaro M, Poli A, Lattanzi F, Visconti A, Nicholas R, Reynolds R. 2019. Iron homeostasis, complement, and coagulation cascade as CSF signature of cortical lesions in early multiple sclerosis. Annals of clinical and translational neurology. 6 (11): 2150-63. doi: 10.1002/acn3.50893.

Miki H, Miura K, Takenawa T. 1996. N-WASP, a novel actin-depolymerizing protein, regulates the cortical cytoskeletal rearrangement in a PIP2-dependent manner downstream of tyrosine kinases. The EMBO journal. 15 (19): 5326-35. doi: 10.1002/j.1460-2075.1996.tb00917.x. Nielsen NM, Rostgaard K, Rasmussen S, Koch-Henriksen N, Storm HH, Melbye M, Hjalgrim H. 2006. Cancer risk among patients with multiple sclerosis: a population-based register study. International journal of cancer. 118 (4): 979-84. doi: 10.1002/ijc.21437.

Padden M, Leech S, Craig B, Kirk J, Brankin B, McQuaid S. 2007. Differences in expression of junctional adhesion molecule- $A$ and $\beta$-catenin in multiple sclerosis brain tissue: increasing evidence for the role of tight junction pathology. Acta neuropathologica. 113 (2): 177-86. doi: 10.1007/s00401-006-0145-x.

Patsopoulos NA, Baranzini SE, Santaniello A, Shoostari P, Cotsapas C, Wong G, Beecham AH, James T, Replogle J, Vlachos I, McCabe C. 2017. The Multiple Sclerosis Genomic Map: Role of peripheral immune cells and resident microglia in susceptibility. BioRxiv. 143933. doi: 10.1101/143933;\%20t.

Perry HV, Cunningham C, Boche D. 2002. Atypical inflammation in the central nervous system in prion disease. Current opinion in neurology. 15 (3): 349-54. doi: 10.1097/00019052200206000-00020. 
393 Plumb J, McQuaid S, Mirakhur M, Kirk J. 2002. Abnormal endothelial tight junctions in active 394 lesions and normal-appearing white matter in multiple sclerosis. Brain pathology. 12 (2): 154-69. 395 doi: 10.1111/j.1750-3639.2002.tb00430.x.

396 Polman CH, O'Connor PW, Havrdova E, Hutchinson M, Kappos L, Miller DH, Phillips JT, 397 Lublin FD, Giovannoni G, Wajgt A, Toal M. 2006. A randomized, placebo-controlled trial of 398 natalizumab for relapsing multiple sclerosis. New England Journal of Medicine. 354 (9): 899 399 910. doi: 10.1056/NEJMoa044397.

400 Polman CH, Reingold SC, Banwell B, Clanet M, Cohen JA, Filippi M, Fujihara K, Havrdova E, 401 Hutchinson M, Kappos L, Lublin FD. 2011. Diagnostic criteria for multiple sclerosis: 2010

402 revisions to the McDonald criteria. Annals of neurology. 69 (2): 292-302. doi:

403 10.1002/ana.22366.

404 Saccone SF, Bolze R, Thomas P, Quan J, Mehta G, Deelman E, Tischfield JA, Rice JP. 2010. 405 SPOT: a web-based tool for using biological databases to prioritize SNPs after a genome-wide 406 association study. Nucleic acids research. 38 (suppl_2): W201-9. doi: 10.1093/nar/gkq513.

407 Sawcer S, Hellenthal G, Pirinen M, Spencer CC, Patsopoulos NA, Moutsianas L, Dilthey A, Su 408 Z, Freeman C, Hunt SE, Edkins S. 2011. Genetic risk and a primary role for cell-mediated 409 immune mechanisms in multiple sclerosis. Nature. 476 (7359): 214. doi: 10.1038/nature10251. 410 Vajkoczy P, Laschinger M, Engelhardt B. 2001. a4-integrin-VCAM-1 binding mediates G 411 protein-independent capture of encephalitogenic T cell blasts to CNS white matter microvessels. 412 The Journal of clinical investigation. 108 (4): 557-65. doi: 10.1172/JCI12440.

413 Wozniak MA, Modzelewska K, Kwong L, Keely PJ. 2004. Focal adhesion regulation of cell 414 behavior. Biochimica et Biophysica Acta (BBA)-Molecular Cell Research. 1692 (2-3): 103-19. 415 doi: 10.1016/j.bbamcr.2004.04.007.

416 Xu Z, Taylor JA. 2009. SNPinfo: integrating GWAS and candidate gene information into 417 functional SNP selection for genetic association studies. Nucleic acids research. 37 (suppl_2):

418 W600-5. doi: 10.1093/nar/gkp290.

419 Yates RL, Esiri MM, Palace J, Jacobs B, Perera R, DeLuca GC. 2017. Fibrin (ogen) and 420 neurodegeneration in the progressive multiple sclerosis cortex. Annals of neurology. 82 (2): 259421 70. doi: 10.1002/ana.24997. 
Figure 1

Genome-wide Manhattan plot showing $-\log _{10}$ of the P-values of 129.547 SNPs (y-axis) against their genomic positions (x-axis) for associations.

The results are plotted left to right from the p-terminal ends of the chromosomes, which are shown in different colors. The blue line represents the P-value threshold (0.05).

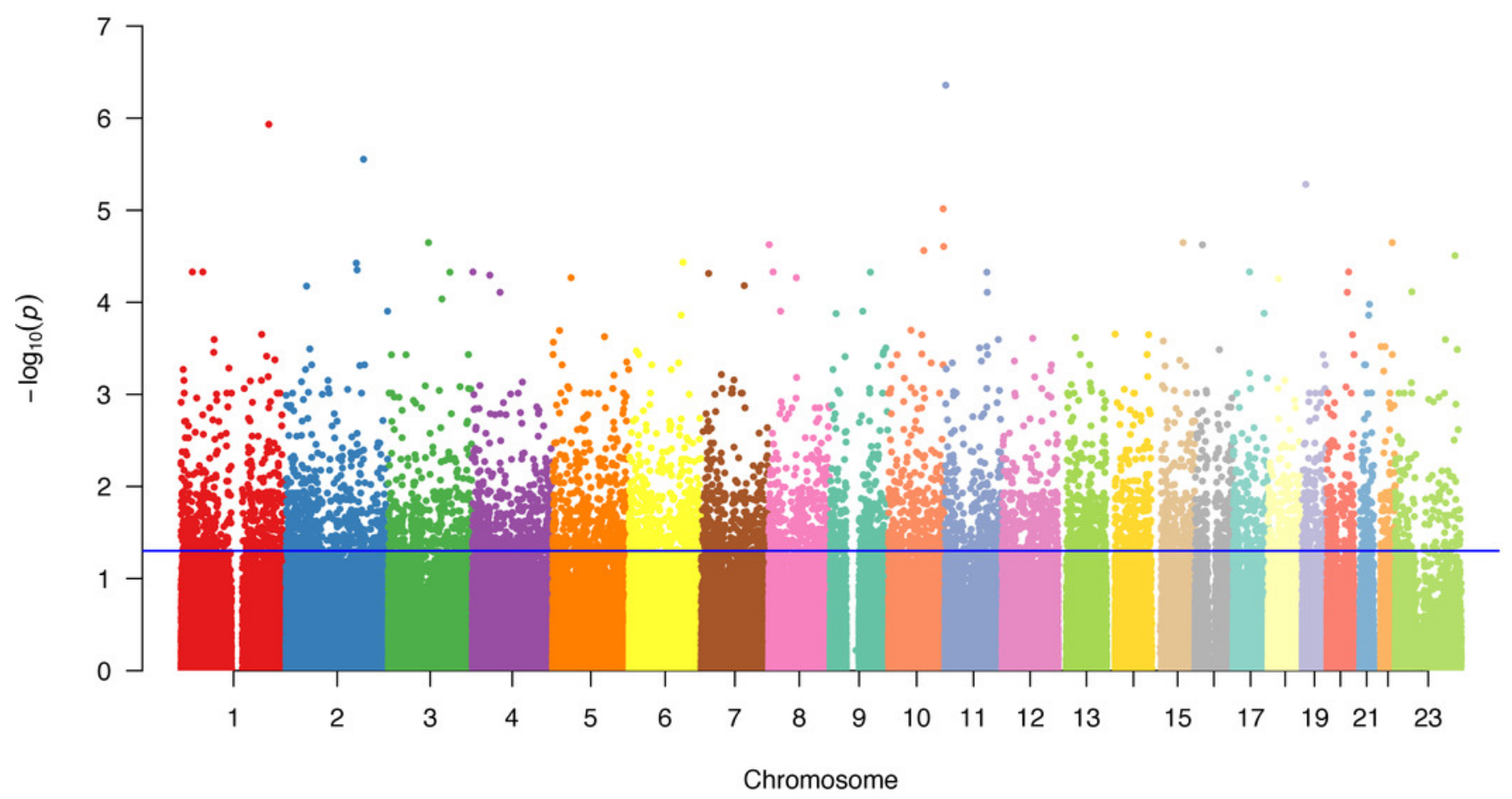




\section{Figure 2}

Clustering dendrogram of the enriched pathways.

The dendrogram shows all 151 enriched pathways, which were grouped in clusters based on their relevance to each other. The red line shows the cut-off for biologically relevant clusters, which was cut at the pairwise distance of 0.55 , resulting in 33 representative clusters. Thirtythree pathways with the lowest P-values in each cluster constituted the representative pathways.

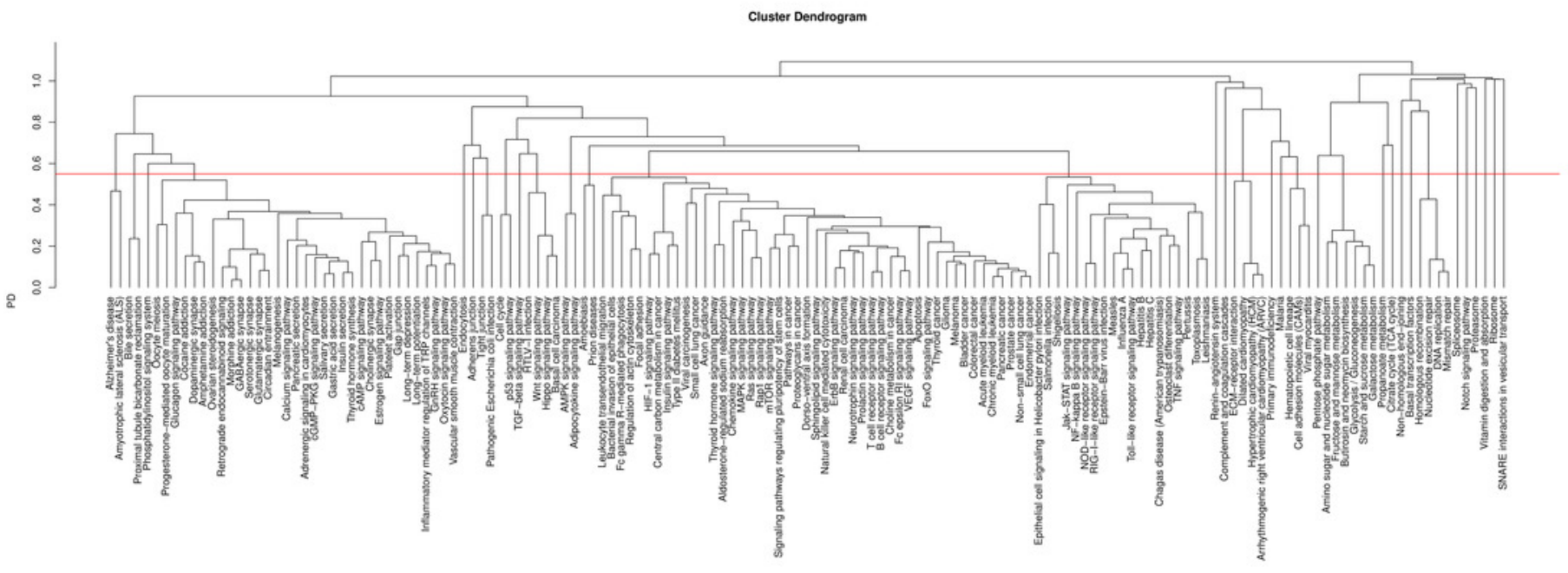


Figure 3

Complement and coagulation cascades.

The illustration of complement and coagulation cascades shows the identified genes by SNP associations (blue) and differentially expressed proteins (yellow) as a representative pathway for the merged data analysis. The pathway was adapted from KEGG: Kyoto Encyclopedia of Genes and Genomes (Kanehisa \& Goto, 2000; hsa04610, 2019) and created with BioRender.com.

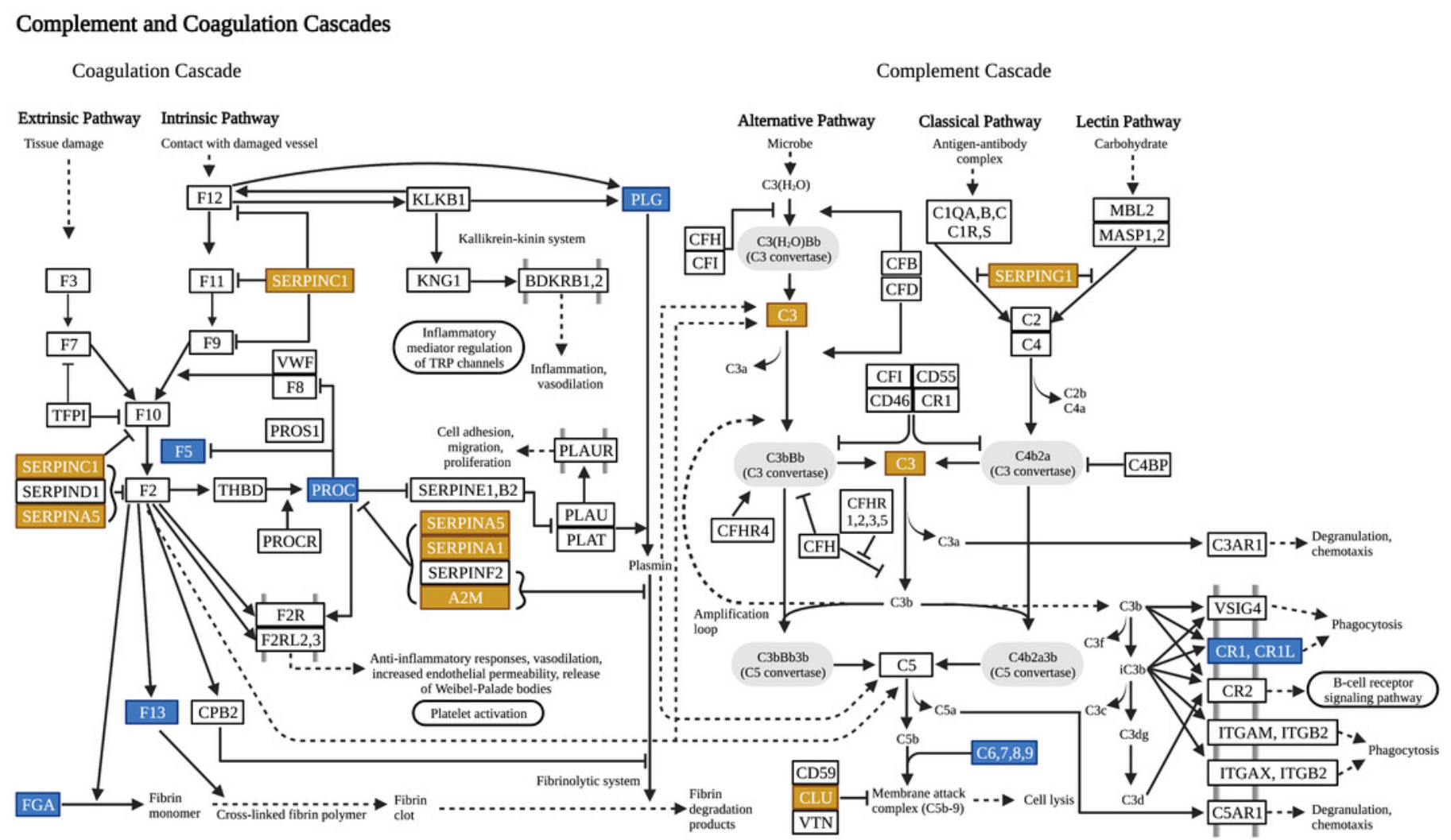




\section{Table $\mathbf{1}$ (on next page)}

The most prominent MS-related pathways emerged from the analyses. 
1

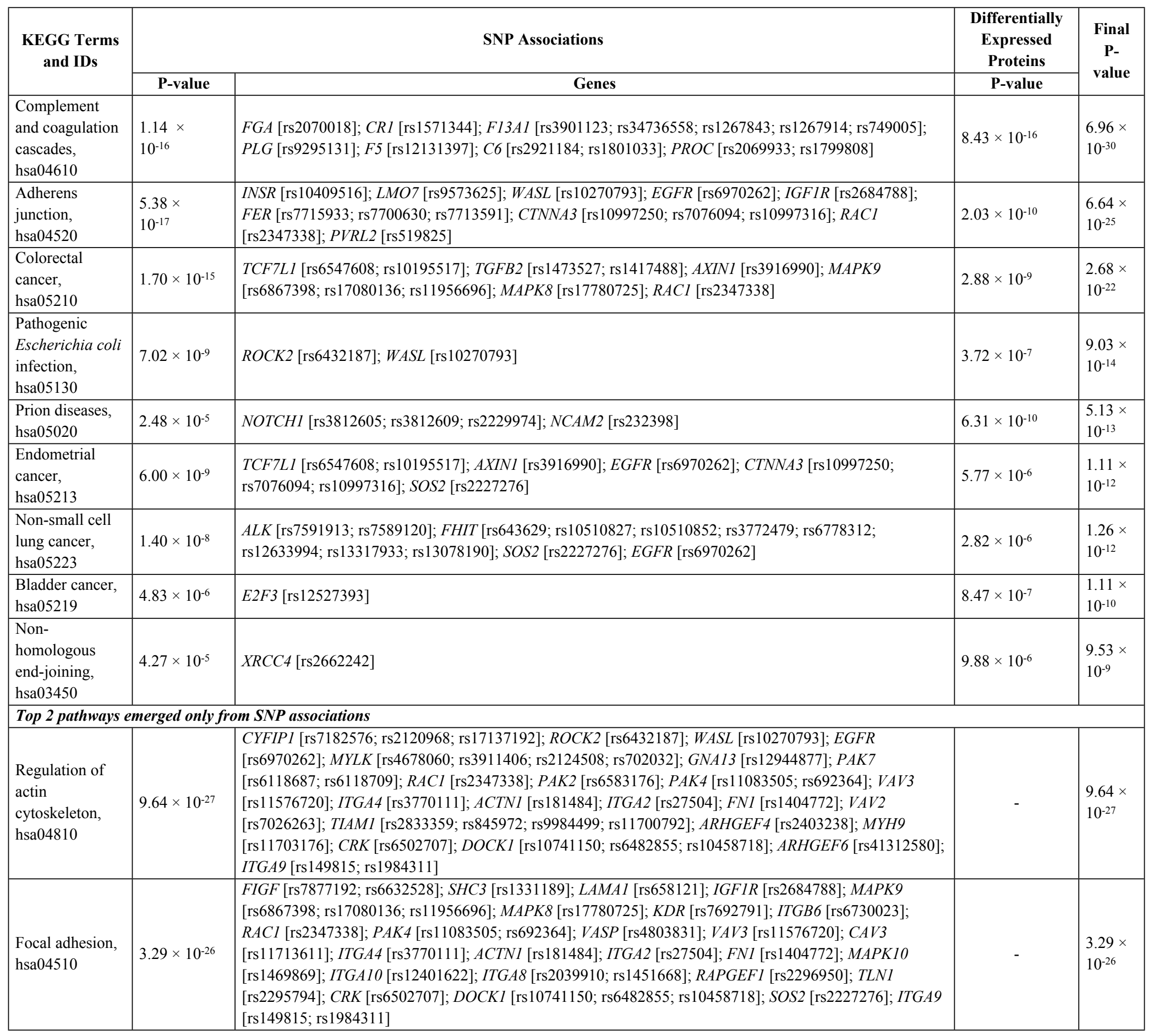


Table 2 (on next page)

Top 5 pathways emerged from each dataset analysis. 


\begin{tabular}{|l|l|}
\hline Top 5 pathways emerged from the proteomic-only data analysis \\
\hline KEGG Terms and IDs & $P$-value \\
\hline Complement and coagulation cascades, hsa04610 & $8.43 \times 10^{-16}$ \\
\hline Adherens junction, hsa04520 & $2.03 \times 10^{-10}$ \\
\hline Prion diseases, hsa05020 & $6.31 \times 10^{-10}$ \\
\hline Colorectal cancer, hsa05210 & $2.88 \times 10^{-09}$ \\
\hline Pathogenic Escherichia coli infection, hsa05130 & $3.72 \times 10^{-07}$ \\
\hline Top 5 pathways emerged from the genomic-only data analysis \\
\hline KEGG Terms and IDs & $P$-value \\
\hline Regulation of actin cytoskeleton, hsa04810 & $9.64 \times 10^{-27}$ \\
\hline Focal adhesion, hsa04510 & $3.29 \times 10^{-26}$ \\
\hline ErbB signaling pathway, hsa04012 & $1.28 \times 10^{-23}$ \\
\hline Rap1 signaling pathway, hsa04015 & $2.35 \times 10^{-20}$ \\
\hline Axon guidance, hsa04360 & $6.36 \times 10^{-20}$ \\
\hline Top 5 pathways emerged from the merged data analysis & \\
\hline KEGG Terms and IDs & $P$-value \\
\hline Complement and coagulation cascades, hsa04610 & $6.96 \times 10^{-30}$ \\
\hline Regulation of actin cytoskeleton, hsa04810 & $9.64 \times 10^{-27}$ \\
\hline Focal adhesion, hsa04510 & $3.29 \times 10^{-26}$ \\
\hline Adherens junction, hsa04520 & $6.64 \times 10^{-25}$ \\
\hline ErbB signaling pathway, hsa04012 & $1.28 \times 10^{-23}$ \\
\hline
\end{tabular}

